\title{
Meta-Learning for Few-Shot Plant Disease Detection
}

\author{
Liangzhe Chen ${ }^{1}$, Xiaohui Cui ${ }^{2}$ DD and Wei $\mathrm{Li}^{1, *(\mathbb{D})}$ \\ 1 School of Artificial Intelligence and Computer Science \& Jiangsu Key Laboratory of Media Design and \\ Software Technology \& Science Center for Future Foods, Jiangnan University, Wuxi 214122, China; \\ lzchen@stu.jiangnan.edu.cn \\ 2 School of Cyber Science and Engineering, Wuhan University, Wuhan 430072, China; xcui@whu.edu.cn \\ * Correspondence: cs_weili@jiangnan.edu.cn
}

Citation: Chen, L.; Cui, X.; Li, W. Meta-Learning for Few-Shot Plant Disease Detection. Foods 2021, 10, 2441. https://doi.org/10.3390/ foods10102441

Academic Editors: António Raposo, Renata Puppin Zandonadi and Raquel Braz Assunção Botelho

Received: 10 September 2021

Accepted: 12 October 2021

Published: 14 October 2021

Publisher's Note: MDPI stays neutral with regard to jurisdictional claims in published maps and institutional affiliations.

Copyright: (c) 2021 by the authors. Licensee MDPI, Basel, Switzerland. This article is an open access article distributed under the terms and conditions of the Creative Commons Attribution (CC BY) license (https:// creativecommons.org/licenses/by/ $4.0 /)$.

\begin{abstract}
Plant diseases can harm crop growth, and the crop production has a deep impact on food. Although the existing works adopt Convolutional Neural Networks (CNNs) to detect plant diseases such as Apple Scab and Squash Powdery mildew, those methods have limitations as they rely on a large amount of manually labeled data. Collecting enough labeled data is not often the case in practice because: plant pathogens are variable and farm environments make collecting data difficulty. Methods based on deep learning suffer from low accuracy and confidence when facing few-shot samples. In this paper, we propose local feature matching conditional neural adaptive processes (LFM-CNAPS) based on meta-learning that aims at detecting plant diseases of unseen categories with only a few annotated examples, and visualize input regions that are 'important' for predictions. To train our network, we contribute Miniplantdisease-Dataset that contains 26 plant species and 60 plant diseases. Comprehensive experiments demonstrate that our proposed LFM-CNAPS method outperforms the existing methods.
\end{abstract}

Keywords: food security; plant disease detection; convolutional neural networks; few-shot; meta-learning

\section{Introduction}

Food shortages may increase in many regions of the world. Coupled with pests and crop failures, food prices have soared. A lot of people may face severe hunger and death. In order to solve the food shortage, it is necessary to ensure the food security and sustainability. Due to pests, diseases [1,2], and lack of horticultural expertise [3-5], food yield loss is greater than $50 \%$ [6]. Food security is increasingly affected by crop production [7]. With the increase of agricultural intensification and the continuous strengthening of the agricultural industry chain, the risks related to viruses and pollution will increase. For the goal of global food security and sustainable development, by 2050, the current demand of crop disease detection needs to increase by $50 \%$ [8].

The traditional method of plant disease detection is manual inspection by farmers or experts. The method of plant disease diagnosis through optical observation of the symptoms on plant leaves incorporates a significantly high degree of complexity [2]. The method laboratory-based such as polymerase chain reaction (PCR), immunofluorescence (IF), and fluorescence in-situhybridization (FISH) require professional laboratory equipment and mass sampling work [9]. Due to this complexity and to the large number of cultivated plants and their existing phytopathological problems, manual plant disease detection can be time-consuming and expensive [10]. By contrast, images under analysis were obtained by employing cameras operating in the visible portion of the electromagnetic spectrum (400-700 nm). In this way, costly equipment or trained personnel are not required for obtaining the input data [11]. Therefore, future users of the developed protocol can acquire data through affordable/cost-effective, portable (thus in situ), and rapid means. With the development of computational systems in recent years, and in particular Graphical Pro- 
cessing Units (GPU) embedded processors, Convolutional Neural Networks (CNNs) [12] is often applied for image classification.

CNNs belong to a stackable feedforward neural network community [12]. The method of image classification through multi-layer CNNs is also called deep learning [13-15]. CNNs have good characterization learning ability, so they are mostly used for feature extraction, and the extracted features have the characteristics of translation invariance. The research on CNNs began in the 1980s and 1990s, and the time delay network and LeNet-5 were the earliest CNNs [12]. For a convolution operation, the essence is a traversal of the convolution kernel on the feature image. The convolution kernel will multiply and add the value at the corresponding position of the input feature image. In recent years, $\mathrm{CNNs}$ have been increasingly incorporated in plant phenotyping concepts. They have been very successful in modeling complicated systems, owing to their ability of distinguishing patterns and extracting regularities from data. Examples further extend to the variety identification in seeds [16] and in intact plants by using leaves [17]. Some research [18] collected and published the datasets of plant diseases that provided data sources for other methods. There are also some works [10] using image segmentation technology to separate the foreground and the background that can further improve the classification accuracy, and also solve the problem of poor performance on the online test.

Although the above deep learning methods have good performance related to plant disease detection, they still have the following problems. The first is the common problem of deep learning; model training requires a large amount of manually labeled datasets. The above-mentioned methods are currently based on the support of a large amount of data. Each category requires more than 1000 pictures. Data collection and marking require manpower and time. There is not enough data to support network training for plant pathogen variables in time, space, and genotype [19]. The second problem is that many of the more than 700 known plant viruses cause devastating diseases and often have wide host ranges. Barley yellow dwarf viruses (BYDV), for example, are distributed worldwide and infect over 150 species of the Poaceae, including most of the staple cereals-wheat, barley, oats, rye, rice, and maize [19]. It is unrealistic to identify all plant diseases at once through one task. However, the emergence of new tasks requires retraining the network. The above methods all limit the total number of categories for specific classification of several plant diseases. Every time a new task is encountered, based on traditional deep learning methods, it is necessary to rearrange the data and train the network to adapt to the task. For different sample numbers and image sizes, professional knowledge is needed to fine-tune the hyperparameters in the network structure. The last problem is the poor interpretability of the method. Compared with manual detection, experts can provide the basis for plant disease detection such as Oval-shaped irregular brown spots appearing on the leaves of plants with rust, and the leaf color on the leaves gradually becoming lighter, and, using fluorescence imaging, temporal and spatial variations of chlorophyll fluorescence were analyzed for precise detection of leaf rust and powdery mildew infections in wheat leaves at $470 \mathrm{~nm}$ [9]. Although the deep-learning-based methods show their effectiveness, it cannot explain their decisions and actions to human users. Therefore, the methods should give visual explanations to illustrate that our approach focuses on diseases' classification. This paper proposed a meta-learning [20] method to solve the challenge of plant disease detection.

Meta-learning is the science of systematically observing how different machine learning approaches perform on a wide range of learning tasks, and then learning from this experience, or meta-data, to learn new tasks much faster than otherwise possible [21]. There is a lot of research on meta-learning. Meta-learning is transfer learning in a broad sense [22], which chooses data from different sources to train the network so that the model has a good classification effect on all kinds of tasks. Few-shot learning [23], which is the problem of making predictions based on a limited number of samples, is an important application direction of meta-learning. The network is trained through other multi-source and sufficient datasets, so that it can deal with the task with few training samples. There is 
a lot of research on few-shot learning. For example, CNAPS [24] and Simple-CNAPS [25] use forward propagation instead of back propagation to solve the problem of overfitting, modular adaptation method [26], and Meta Fine-Tuning [27], which is also called CrossDomain Few-Shot learning, can be trained to perform both tasks across domains. There is also some research on metric learning [28] such as MatchingNet [29] and ProtoNet [23] to solve the problem of insufficient samples and the poor performance of the classifier. Although there have been many works on few-shot learning, most of the works are more theoretically focused, and do not focus on specific applications. Based on the previous work, this paper applied meta-learning to plant diseases detection.

\section{Materials and Methods}

In this section, initially, the datasets chosen for training and testing are introduced. Afterwards, the meta-learning method proposed for plant detection called LFM-CNAPS is presented. Finally, visual explanations technology called TAM is introduced.

\subsection{Datasets}

The key to few-shot plant disease detection lies in the generalization ability of the pertinent model when presented with novel disease categories. Thus, high-diversity datasets are necessary for training the model that can detect unseen plant diseases. In this paper, Meta-Dataset [30] and Miniplantdisease-Dataset are chosen for model training.

A Meta-Dataset [30] is composed of 10 public datasets including ILSVRC-2012 (ImageNet) [31], Omniglot [32], FGVC-Aircraft (Air-craft) [33], CUB-200-2011 (Birds) [34], Describable Textures (DTD) [35], QuickDraw [36], FGVCx Fungi (Fungi)[37], VGG Flower (Flower) [38], Traffic Signs (Signs) [39], and MSCOCO [40]. Meta-Dataset is comprised of multiple existing datasets that contains more than 110,000 few-shot classification tasks. The tasks span a variety of visual concepts (natural and human-made) and vary in how fine-grained the class definition is [30]. Through Meta-Dataset training, the models' ability to leverage diverse training sources will be improved.

Miniplantdisease-Dataset proposed in this paper is composed of Apple foliar disease Dataset [41] and PlantVillage-Dataset [18]. The Apple foliar disease Dataset contains 3651 high-quality and real photos of various apple foliar diseases. The PlantVillageDataset has released more than 50,000 specialized images through the online platform Plantvillage [18]. The PlantVillage-Dataset contains various diseases per plant categories, while the Apple foliar disease Dataset only contains healthy and unhealthy two labels per plant categories. In keeping with a results report, the PlantVillage is divided into two parts with a ratio of 8:2 to compose Miniplantdisease-Dataset and test model, respectively. We report results using 48 plant diseases for training called in-domain MiniplantdiseaseDataset, reserving other 12 plant diseases for out-of-domain performance evaluation.

\section{2. $L F M-C N A P S$}

The method proposed in this paper to solve few-shot plant disease recognition is local feature matching conditional neural adaptive processes (LFM-CNAPS). As shown in Figure 1, it contains four main parts: input task, conditional adaptive feature extractor, and local feature matching classifier and parameters optimizer.

\subsubsection{Task}

Miniplantdisease-Dataset contains many different meta-tasks. The model learns the generalization ability from meta-tasks. When facing new categories, the classification can be completed without changing the existing model. The Miniplantdisease-Dataset contains 60 plant disease categories, with multiple samples in each category. For any meta-task in Miniplantdisease-Dataset, five plant disease categories will be randomly selected, with five samples for each category (a total of 25 samples). These samples with their labels will be constructed as support images and support labels of meta-task. In addition, then extract 50 samples for test from the remaining five categories samples as query images and query 
labels. That is, the model is required to learn how to distinguish these five categories from 25 samples. Such a task is called a 5-way 5-shot problem.

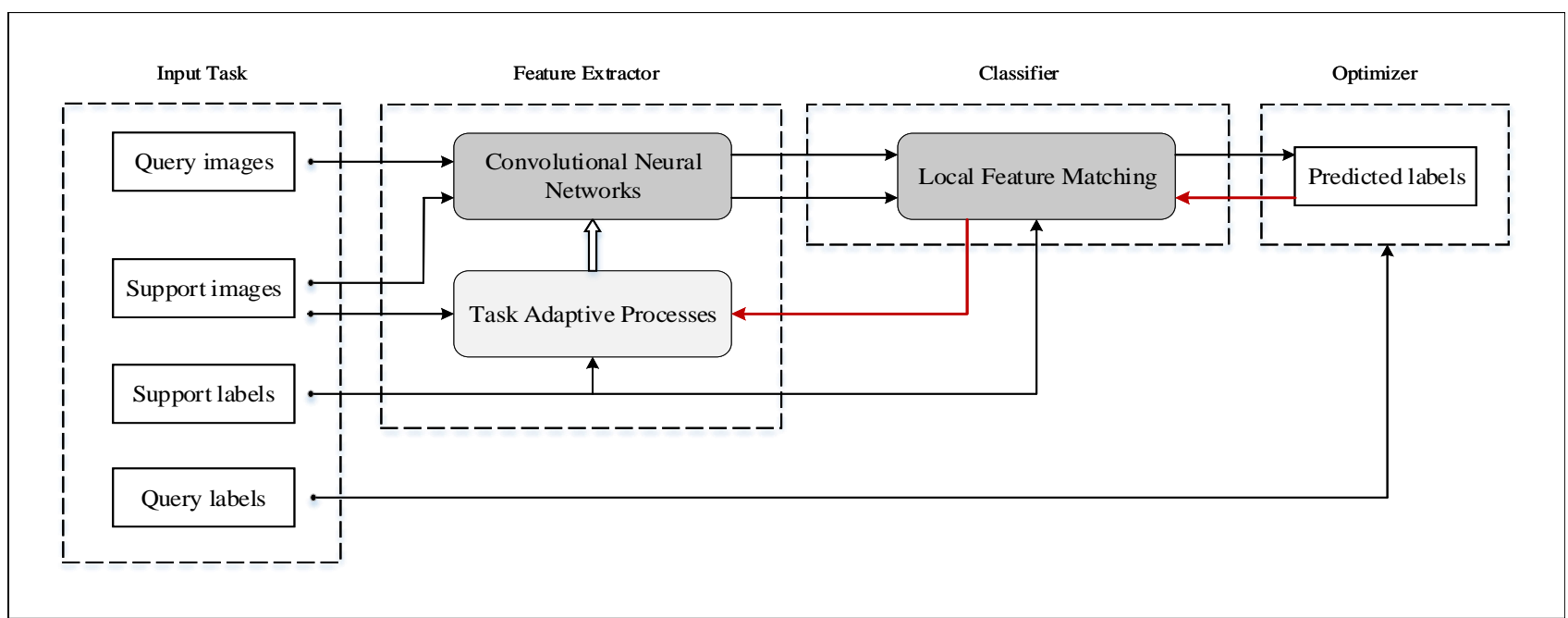

Figure 1. Flow chart of LFM-CNAPS. In the figure, the white solid squares are data variable, the black arrows are the data flow direction, the white hollow arrows are forward propagation, and the red arrows are backward propagation.

\subsubsection{Conditional Adaptive Feature Extractor}

The feature extractor chosen in this paper consists of two parts, a CNN framework named RESNET18 [42] and task adaptive processes [24]. Among them, RESNET18 is a stackable CNN layer with a batch normalization layer to prevent vanishing gradient and exploding gradient. The task adaptive process [24] is an effective method of impacting CNN intermediate variables to adapt the task [43]. The core of the task adaptive process is to choose forward propagation instead of back propagation to prevent overfitting due to few samples.

RESNET18 mainly contains CNNs and a Batch Normalization layer. The CNNs are essentially to do a dot product between the filter and the local area of the input data. The convolution kernel will multiply and add the value at the corresponding position of the input data, as shown in (1):

$$
\operatorname{Con}\left(I_{x, y}, K\right)=\sum_{c=1}^{C} \sum_{h=1}^{H} \sum_{w=1}^{W} I_{x+h, y+w, c} \times K_{h, w}
$$

Among them, I represents the input data, $K$ represents the convolution kernel, and $x$ and $y$ represent the position of the convolution kernel on the feature map I. $H, W$, and $C$ respectively represent the length and width of the convolution kernel and the number of channels. The CNNs will extract specific local features according to the convolution kernel parameters. For CNNs, there are many hyperparameters such as the size of the convolution kernel, sliding step size, and the number of CNN layers. Different hyperparameter settings will have a great impact on the accuracy of the model. RESNET18 gives the hyperparameters suitable for most image feature extractions [42]. The core of RESNET18 [42] is stackable CNN layers, and the hyperparameters of the CNNs are fixed. On this basis, Batch Normalization layer is applied. In the Batch Normalization layer, such an operation is shown in (2):

$$
H(X)=F(X)+X
$$

Among them, $H(X)$ represents the Batch Normalization operation, $F(X)$ represents the corresponding CNNs, and $X$ represents the input. It can be seen intuitively that the process of Batch Normalization layer is to add the input $X$ and the result of the CNNs. Batch Normalization effectively solves the problem of gradient vanishing and degradation 
caused by the network being too deep. Batch Normalization layers make deep network training possible.

The task adaptive process contains the task encoder and FILM layer [44]. The task encoder is composed of CNN layers and fully connected layers that take the support set as input and FILM layer parameters as output. The task encoder provides FILM layer parameters to make CNNs better adapt tasks. For traditional deep learning methods, back propagation is an important method of updating parameters. However, most deep learning methods require a large number of labeled samples. For a few-shot task, there are only a few labeled samples for training. Too few samples to update the parameters through back propagation will cause overfitting. That is, the accuracy of the training set is very high, and the result of the test set is very poor. To avoid this, the FILM layer [44] is proposed to perform affine transformation on the intermediate features of the CNNs, as shown in (3):

$$
\sum_{c=1}^{C} \sum_{x=1}^{X} \sum_{y=1}^{Y} I_{x, y, c} \times \gamma_{c}+\beta_{c}
$$

Among them, $I$ is the middle feature map, and $X, Y$, and $C$ represent the length and width of the feature and the number of channels. $\gamma$ and $\beta$ are the parameters of the FILM layer and they are generated by the task encoder. The parameters updated by back propagation are proportional to the volume of the convolution kernel, and the forward propagation only needs to update the parameters that are proportional to the number of channels. Therefore, the depth of the overall network has not changed, but the number of updated parameters are reduced, avoiding the overfitting caused by few samples.

\subsubsection{Local Feature Matching Classifier}

For image classification methods, classifiers are indispensable [45]. For the traditional method, after handcrafted features are extracted, a separate classifier such as SVM is needed. For deep learning, a fully connected layer and activation function are generally chosen as a classifier. For SVM, the parameters need to be trained separately [46]. For deep learning classifier that can be trained end-to-end, the fully connected layer contains hundreds of parameters that need to be optimized. When samples are not enough, parameter optimization can be difficult. Therefore, this paper chooses metric learning as the classifier for few-shot plant disease detection.

The obvious advantage of the metric learning classifier is that there are no parameters be optimized. For metric learning, the distance between the feature value and the prototype [23] is calculated to determine which category the query sample belongs to. The concept of prototype comes from the prototype network [23], and the most common definition of prototype is the average of each category's features. To output the labels of query set, methods usually calculate the metric distance between query set and each prototype.

This paper chooses the local feature matching classifier [47]. This method has two advantages. First, for other metric learning methods, the extracted features need to be pooled that destroy the original spatial information of the features. The local feature matching classifier directly takes the extracted high-dimensional features for classification. Secondly, the local feature matching method can reduce the impact of occlusion or noise on classification to a certain extent that improves the robustness of the algorithm. The calculation process of the local feature matching classifier is as follows, as shown in (4):

$$
\sum_{x 1=1}^{W} \sum_{y 1=1}^{H} \operatorname{Max}_{K}\left(\left\{\frac{F_{x 1, y 1}^{q} \cdot F_{x 2, y 2}^{c}}{\left|F_{x 1, y 1}^{q}\right| \times\left|F_{x 2, y 2}^{c}\right|} \mid 1 \leqslant x 2 \leqslant W, 1 \leqslant y 2 \leqslant H\right\}\right)
$$

Among them, $F^{q}$ and $F^{c}$ respectively represent the feature of query set and the prototype, and $H$ and $W$ are the length and width of the feature map. $\operatorname{Max}_{K}()$ represents the function that is to select top $K$ maximums. The classifier regards each pixel of the feature map as a local feature of the image. The calculation process of (4) is to traverse all local 
features on $F^{q}$. Calculate the cosine distance between the local features on $F^{q}$ and all the local features on $F^{c}$. The maximum $K$ values summation is selected as the matching value, and the final summation of all matching values is the metric distance between $F^{q}$ and $F^{c}$. The larger the metric distance value represents, the closer $F^{q}$ and $F^{c}$ is. When all the category prototypes are traversed, the category with the largest metric value is selected as the category of the query image $F^{q}$.

\subsubsection{Parameters Optimizer}

The meta-learning method chosen in this paper contains the following parameters: the CNN parameters in RESNET18, the task adaptive encoder parameters, and the FILM layer parameters. Among them, the parameters of RESNET18 are pre-trained and do not participate in the update, and the parameters of the FILM layer are generated by the task encoder. Therefore, for the LFM-CNAPS, it is the parameters in the task adaptive encoder that need to be trained and updated. The parameter update is reflected in two parts. First, during the meta-training process, the parameters in the task adaptive encoder are updated through back propagation. Secondly, during the meta-test process, the parameters in the FILM layer are updated through forward propagation. The parameters optimizer is proposed for back propagation.

For the optimizer, the most important thing is the loss function and optimization method. The loss function and optimization algorithm chosen in this paper are cross entropy loss [48] and Adam algorithms [49]. The cross entropy loss is calculated as (5):

$$
\sum y_{c} \log \left(p_{c}\right)
$$

where $y$ represents the category label, $c$ represents the category name, and $p$ represents the predicted probability. If the query image this time is of category $c$, then the value of $y_{c}$ is 1 ; otherwise, it is 0 . For the prediction result of the algorithm, various probabilities $p_{c}$ are obtained through the sigmoid activation function. In summary, the cross-entropy loss obtains a loss value from the label predicted by the model and the actual label.

\subsection{Task Activation Mapping}

For deep learning, most algorithms are black box. They reduce the loss through back propagation and improve the test accuracy through a large number of samples. However, deep models are not easy to visualize and could not give the basis of classification results. For CNNs, there have been many studies on visual explanations [50].

The TAM algorithm proposed in this paper is modified on the basis of the GradCAM [50]. The Grad-CAM process is as follows: first, a test image is needed as input, and the classification probability is obtained through the trained network. Grad-CAM will select the channel where the back propagation is located through the label. When the back propagation reaches the last layer of the CNNs, Grad-CAM would record the parameter gradient of the last layer. The gradient tensor will be averaged in the channel direction, and a one-dimensional variable whose length is the number of channels will be obtained. Grad-CAM would multiply the one-dimensional variable with the intermediate variable of the last layer to obtain the activated intermediate variable. The intermediate variables will be averaged in the direction of the feature map to obtain a two-dimensional activation layer. Grad-CAM will convert the two-dimensional activation layer mapping from 0 to 255 into a heat map. The heat map will be mapped to the input image to get a visual CNN heat map. Grad-CAM obtains the influence of various features by the degree of the convolution gradient. The brighter the red in the figure, the greater this part of the feature effect on the result.

However, for the method in this paper, the use of Grad-CAM has been restricted. Since the classifier does not contain parameters, the back propagation starts directly from the last layer of the CNNs and the parameters updated by back propagation are part of the task encoder. TAM is proposed based on the Grad-CAM. It can be known from the Grad-CAM that the pooled gradient one-dimensional variable needs to be obtained, and, from (3), 
$\gamma$ generated by the encoder is such a variable. For the task encoder, its function is to generate parameters through task features, and interfere with the intermediate variables of RESNET18. Therefore, the $\gamma$ of the last layer of CNNs is chosen to average the intermediate variables in the direction of the feature map to obtain the two-dimensional activation layer. Then, TAM will perform its mapping to get the CNN heat map. Compared with Grad-CAM, TAM does not choose categories for gradient transformation but task features. Secondly, Grad-CAM is done through back propagation gradients and TAM is through forward propagation. Visual explanations of tomato disease output by TAM are shown in Figure 2.

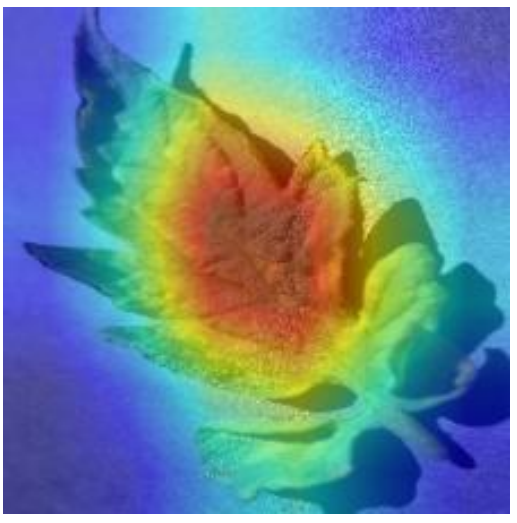

(a)

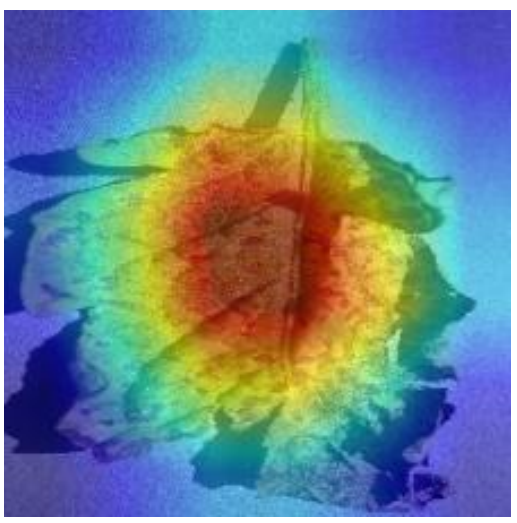

(c)

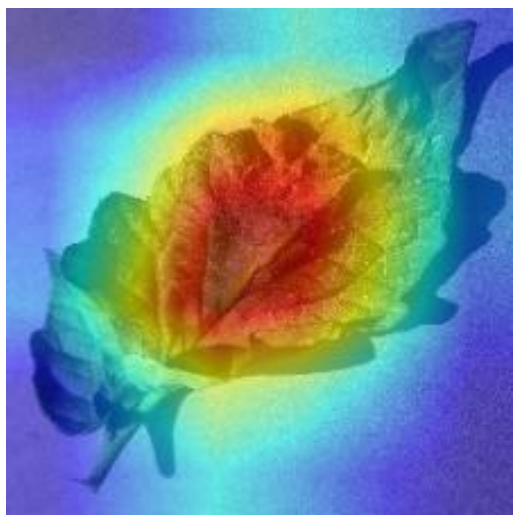

(b)

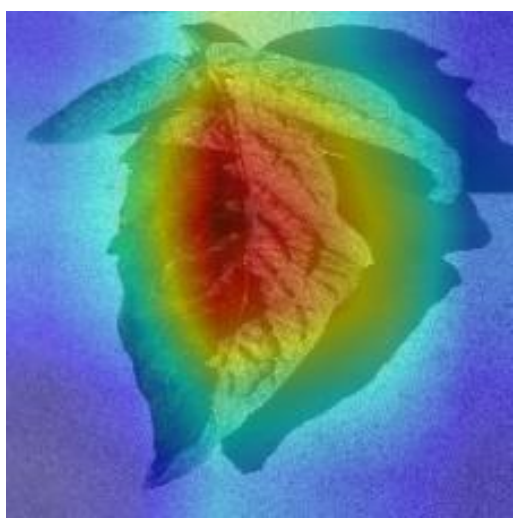

(d)

Figure 2. Visual explanations of tomato disease. (a) Leaf mold; (b) Late blight; (c) Septoria leaf spot; (d) two spotted spider mite.

\section{Results}

We evaluate LFM-CNAPS on the Miniplantdisease-Dataset family of datasets, demonstrating improvements by ablation experiment. Two prediction visual explanations are also given.

\subsection{Performance of Plant Disease Detection}

We train LFM-CNAPS on the Meta-Dataset and Miniplantdisease-Dataset, evaluate it on the Miniplantdisease-Dataset and PlantVillage-Dataset. To investigate the performance of LFM-CNAPS proposed, six comparison algorithms are adopted. First, the deep learning method [2] composed of CNNs and a fully connected layer is chosen (RESNET18 + FC). To control variables, CNNs use RESNET18 that is the same as LFM-CNAPS. Next, the model composed of RESNET18 and local feature matching classifier is chosen (RESNET18 + LFM) to show the effect of task adaptive processes of LFM-CNAPS. Finally, four few-shot learning methods are adopted including: MatchingNet [29], ProtoNet [23], Simple-CNAPS [25], and Meta Fine-Tuning [27]. The information of machine specifications and time cost is shown in Table 1. 
Table 1. Machine specifications and time cost.

\begin{tabular}{cc}
\hline Name & Value \\
\hline Video Memory & $11 \mathrm{G}$ \\
Graphics & NVIDIA GeForce GTX 1080 Ti \\
Processor & Intel(R) Xeon(R) CPU E5-2640 \\
Operating system & Windows 10 Home 64 \\
Training time & $17,548.73 \mathrm{~s}$ \\
Test time & $57.36 \mathrm{~s}$ \\
\hline
\end{tabular}

The training results of the Meta-Dataset are shown in Table 2. Table 2 is the result of the method performance on Meta-Dataset which was also trained on Meta-Dataset. Among them, cifar10 and cifar100 are not included in the training dataset and are only used for testing. The results in Table 2 can reflect the performance of the approach on general classification tasks. These include classification of animal species, classification of objects and tools, and classification of handwritten fonts. The task format of the Meta-Dataset is not fixed. Through 110,000 times of training, the algorithm has a better classification effect.

Table 2. LFM-CNAPS test accuracy on the Meta-Dataset.

\begin{tabular}{cc}
\hline Dataset Name & Accuracy (\%) \\
\hline ilsvrc 2012 & $55.0+/-1.0$ \\
omniglot & $92.0+/-0.6$ \\
aircraft & $82.4+/-0.6$ \\
cu birds & $74.3+/-0.8$ \\
dtd & $65.3+/-0.7$ \\
quickdraw & $75.5+/-0.8$ \\
fungi & $48.0+/-1.1$ \\
Vgg flower & $89.4+/-0.5$ \\
Traffic sign & $68.2+/-0.7$ \\
mscoco & $51.1+/-1.0$ \\
mnist & $93.3+/-0.4$ \\
cifar10 & $71.1+/-0.7$ \\
cifar100 & $57.3+/-1.0$ \\
\hline
\end{tabular}

The training results of the in-domain Miniplantdisease-Dataset are shown in Table 3. A total of 20,000 tasks were randomly generated, including 60 types of plant diseases. The names of various types of plants, the number of their diseases, and the number of corresponding samples are declared in the table. The data sources are distinguished in the table. It can be seen that the two datasets do not contain the same plant categories. Each plant of Apple foliar disease has only two categories: healthy and diseased. The number of plant diseases in PlantVillage is relatively random, as many as 10 and as few as one. Through cross-domain dataset training, the model can be more robust. Secondly, from the perspective of sample size, the sample size of Apple foliar disease is much smaller than that of PlantVillage. The unbalanced sample distribution is more practical for application because there is no absolutely balanced sample in reality, and most plant classification samples are random. From the results, after 20,000 trainings, the average accuracy of the algorithm reached $97.5 \%$. 
Table 3. LFM-CNAPS train results on in-domain Miniplantdisease-Dataset.

\begin{tabular}{|c|c|c|}
\hline Species & Number of Plant Diseases & Number of Samples \\
\hline \multicolumn{3}{|c|}{ Apple foliar disease } \\
\hline Alstonia Scholaris & 2 & 433 \\
\hline Arjun & 2 & 452 \\
\hline Bael & 2 & 266 \\
\hline Chinar & 2 & 223 \\
\hline Gauva & 2 & 419 \\
\hline Jamun & 2 & 624 \\
\hline Jatropha & 2 & 257 \\
\hline Lemon & 2 & 236 \\
\hline \multicolumn{3}{|c|}{ PlantVillage } \\
\hline Apple & 4 & 7169 \\
\hline Blueberry & 1 & 1816 \\
\hline Cherry & 2 & 3509 \\
\hline Corn & 4 & 7316 \\
\hline Grape & 4 & 7222 \\
\hline Orange & 1 & 2010 \\
\hline Peach & 2 & 3566 \\
\hline Pepper & 2 & 3901 \\
\hline Potato & 2 & 3763 \\
\hline Tomato & 10 & 18,345 \\
\hline \multicolumn{2}{|c|}{ Number of training steps } & Training accuracy (\%) \\
\hline \multirow{2}{*}{\multicolumn{2}{|c|}{$\begin{array}{l}10,000 \\
20,000\end{array}$}} & 97.0 \\
\hline & & 97.5 \\
\hline
\end{tabular}

Table 4 shows the test results of out-of-domain Miniplantdisease-Dataset. Out-ofdomain and in-domain datasets do not contain the same plant diseases. Testing the algorithm through untrained plant diseases can better reflect the robustness of the algorithm. The accuracy of algorithms for newly emerged plant diseases is also more practical. The out-of-domain datasets include 12 plant disease categories and 600 random tasks. LFM-CNAPS has an average accuracy rate of $93.3 \%$ on out-of-domain dataset.

Table 5 shows ablation studies of LFM-CNAPS on an Out-of-Domain MiniplantdiseaseDataset. Our model mainly includes conditional adaptive feature extractor and local feature matching classifier components. A conditional adaptive feature extractor extracts meaningful features via forward propagation, which helps the model learn those features even in the few-shot dataset. A local feature matching classifier replaces the fully connected layers with metric learning to avoid overfitting which deeply hurts the performance of the neural network. The result of ablation experiments is shown in Table 5. LFM-CNAPS with only conditional adaptive feature extractor holds $86.1 \%$ accuracy and LFM-CNAPS with only a local feature matching classifier reaches $85.2 \%$ accuracy. However, LFM-CNAPS with the two components has $93.9 \%$ accuracy.

The test results of PlantVillage are shown in Table 6, which contains a total of 38 plant diseases. Although some plant disease categories in PlantVillage are used for meta-training, the test pictures are different from the training pictures. The number of categories and the number of samples are more than the first test. Therefore, the test on PlantVillage is more challenging than the first test, and the average accuracy of LFM-CNAPS is $89 \%$. 
Table 4. LFM-CNAPS test results on an Out-of-Domain Miniplantdisease-Dataset.

\begin{tabular}{cc}
\hline Plant State & Number of Samples \\
\hline Mango diseased & 265 \\
Mango healthy & 170 \\
Pomegranate diseased & 272 \\
Pomegranate healthy & 287 \\
Pongamia Pinnata diseased & 276 \\
Pongamia Pinnata healthy & 322 \\
Potato Late blight & 1939 \\
Raspberry healthy & 1781 \\
Soybean healthy & 2022 \\
Squash Powdery mildew & 1736 \\
Strawberry healthy & 1824 \\
Strawberry Leaf scorch & 1774 \\
\hline Method & Accuracy (\%) \\
\hline RESNET18 + FC & $20.0+/-0.5$ \\
MatchingNet & $19.5+/-0.5$ \\
ProtoNet & $20.5+/-0.6$ \\
RESNET18+ LFM & $85.2+/-0.7$ \\
Simple-CNAPS & $92.5+/-0.4$ \\
Meta Fine-Tuning & $91.14+/-0.5$ \\
LFM-CNAPS & $93.9+/-0.4$ \\
\hline
\end{tabular}

Table 5. Ablation studies of LFM-CNAPS on an Out-of-Domain Miniplantdisease-Dataset.

\begin{tabular}{ccc}
\hline Feature Extractor & Classifier & Accuracy (\%) \\
\hline & & $20.0+/-0.5$ \\
$\checkmark$ & & $86.1+/-0.6$ \\
$\checkmark$ & $\checkmark$ & $85.2+/-0.7$ \\
& $\checkmark$ & $93.9+/-0.4$ \\
\hline
\end{tabular}

Table 6. LFM-CNAPS test results on a PlantVillage-Dataset.

\begin{tabular}{|c|c|c|}
\hline Plant Category & Number of Plant Diseases & Number of Samples \\
\hline Apple & 4 & 1943 \\
\hline Blueberry & 1 & 454 \\
\hline Cherry & 2 & 877 \\
\hline Corn & 4 & 1829 \\
\hline Grape & 4 & 1805 \\
\hline Orange & 1 & 503 \\
\hline Peach & 2 & 891 \\
\hline Pepper & 2 & 975 \\
\hline Potato & 3 & 1426 \\
\hline Raspberry & 1 & 445 \\
\hline Soybean & 1 & 505 \\
\hline Squash & 1 & 434 \\
\hline Strawberry & 2 & 900 \\
\hline Tomato & 10 & 4585 \\
\hline \multicolumn{2}{|c|}{ Method } & Test accuracy (\%) \\
\hline \multicolumn{2}{|c|}{ RESNET18 + FC } & $19.8+/-0.5$ \\
\hline \multicolumn{2}{|c|}{ RESNET18 + LFM } & $81.7+/-0.7$ \\
\hline \multicolumn{2}{|c|}{ LFM-CNAPS } & $89.0+/-0.5$ \\
\hline
\end{tabular}




\subsection{Visual Explanations}

Abnormal phenotype can be caused by either abiotic or biotic stress. The former is caused, for instance, by lack or excess of nutrients or water [51]. The latter can be caused by fungi, bacteria, and viruses. The typical symptomatology of (abiotic or biotic) stress includes discoloration, necrosis, decay, wilting, and atypical forms. Most of the existing deep learning methods for plant disease classification pay more attention to the test accuracy, and do not pay much attention to the classification basis. However, for practical application, a reasonable classification basis is more convincing and more acceptable. While our method gives the classification results, it also can save the classification's heat map by TAM technology. As shown in Figure 3, (a) is a sample map of Alstonia Scholari affected by pests, and (b) is a sample map of potato with late blight. The red part is the part that the algorithm pays more attention to, and it is also the core part that affects the classification result. For (a), the red part mainly appears near the wormhole, and, for (b), the red part is also where the leaves turn yellow and wither. Although it is impossible to give a detailed description of the causes of plant diseases, the focus of a heat map can be used to visualize the parameters of the CNNs, and the interpretability of the black box network can be improved to a certain extent.

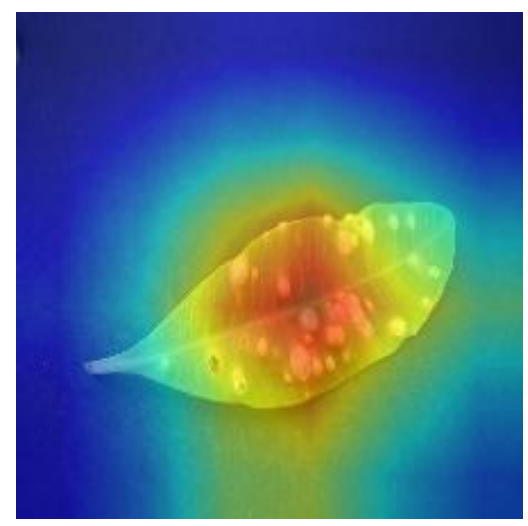

(a)

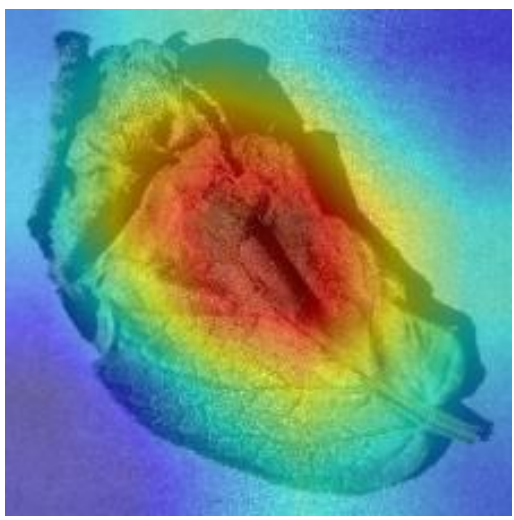

(b)

Figure 3. LFM-CNAPS classification basis is visualized by TAM. (a) the sample of diseased Alstonia Scholari; (b) the sample of potato with late blight.

\section{Discussion}

Pests and diseases seriously threaten crop yields, leading to food shortages, e.g., more than 800 million people do not have adequate food; 1.3 billion live on less than $\$ 1$ a day and at least $10 \%$ of global food production is lost to plant disease [19]. In order to combat the losses, the emerging plant disease needs to be detected before it has a largescale impact on crop growth. This paper intends to propose a method that can detect plant diseases with few samples. The results showed that LFM-CNAPS proposed has an average accuracy of $93.9 \%$ on detecting unseen plant disease with only 25 annotated examples. The method RESNET18 + FC based on deep learning [10] only has an accuracy of $20.0 \%$. More specifically, when classifying the five diseases of tomato: bacterial spot, early blight, healthy, late blight, and leaf mold, the performance of LFM-CNAPS is much better than the other two methods. LFM-CNAPS can give visual explanations through TAM, similar to optical observation of the symptoms on plant leaves. As show in Figure 3, Alstonia Scholari's wormhole is presented. However, due to complexity, even experienced agronomists often fail to successfully diagnose specific diseases [2]. For example, it is difficult for people to distinguish the corn with northern leaf blight from the corn with gray leaf spot. LFM-CNAPS adaptively adjusts and extracts the potential differences between the two categories by task adaptive processes. This feature will be more abstract rather than simple geometric features. On a commercial scale, evidently, a capital investment is initially required for adopting the employed approach [52]. Nevertheless, the wide- 
ranging large-scale commercial applications can provide high returns through considerable improvements in process enhancement and cost reduction.

Limitations of the study are due to a single task format. All tests are based on using 25 samples for plant disease detection. Recommendations for further research are to deal with a different scale of samples for better classification results.

\section{Conclusions}

This paper proposed LFM-CNAPS to solve few-shot plant disease detection and made the following contributions: first, a Miniplantdisease-Dataset suitable for meta-learning is provided, including two public datasets, 60 plant disease categories. Secondly, the LFM-CNAPS proposed is evaluated on the Miniplantdisease-Dataset, with an accuracy rate of $93.9 \%$. Finally, TAM was proposed for CNN visualization. Without affecting the classification results and time cost, the classification heat map is saved to realize visual explanations.

Author Contributions: W.L. contributed to the conceptualization design and the design of the methodology. L.C. contributed to the algorithm achievement and paper writing. X.C. provides the data and revises this paper. All authors have read and agreed to the published version of the manuscript.

Funding: This work was supported by the Fundamental Research Funds for the Central Universities (No. JUSRP121073, JUSRP521004).

Institutional Review Board Statement: Not applicable.

Informed Consent Statement: Not applicable.

Data Availability Statement: The dataset can be obtained from https:/ /www.kaggle.com/vipoooool/ new-plant-diseases-dataset (accessed on 14 July 2021) and https://www.kaggle.com/c/plantpathology-2020-fgvc7 (accessed on 14 July 2021) .

Conflicts of Interest: The authors declare no conflict of interest.

\section{References}

1. Muimba-Kankolongo, A. Food Crop Production by Smallholder Farmers in Southern Africa II Climates and Agroecologies; Academic Press: Cambridge, MA, USA, 2018; pp. 5-13.

2. Ferentinos, K.P. Deep learning models for plant disease detection and diagnosis. Comput. Electron. Agric. 2018, 145, 311-318. [CrossRef]

3. Mohameth, F.; Bingcai, C.; Sada, K.A. Plant disease detection with deep learning and feature extraction using plant village. J. Comput. Commun. 2020, 8, 10-22. [CrossRef]

4. Klauser, D. Challenges in monitoring and managing plant diseases in developing countries. J. Plant Dis. Prot. 2018, 125, $235-237$. [CrossRef]

5. Kader, A.A.; Kasmire, R.F; Reid, M.S.; Sommer, N.F.; Thompson, J.F. Postharvest Technology of Horticultural Crops; University of California Agriculture and Natural Resources: Davis, CA, USA, 2002.

6. Teng, P.S.; James, W.C. Disease and yield loss assessment. In Plant Pathologists Pocketbook; CABI: Wallingford, UK, 2002.

7. Maxwell, S. Food security: A post-modern perspective. Food Policy 1996, 21, 155-170. [CrossRef]

8. Hunter, M.C.; Smith, R.G.; Schipanski, M.E.; Atwood, L.W.; Mortensen, D.A. Agriculture in 2050: Recalibrating targets for sustainable intensification. Bioscience 2017, 67, 386-391. [CrossRef]

9. Fang, Y.; Ramasamy, R.P. Current and prospective methods for plant disease detection. Biosensors 2015, 5, 537-561. [CrossRef] [PubMed]

10. Sharma, P.; Berwal, Y.P.S.; Ghai, W. Performance analysis of deep learning CNN models for disease detection in plants using image segmentation. Inf. Process. Agric. 2020, 7, 566-574. [CrossRef]

11. Taheri-Garavand, A.; Nejad, A.R.; Fanourakis, D.; Fatahi, S.; Majd, M.A. Employment of artificial neural networks for noninvasive estimation of leaf water status using color features: A case study in Spathiphyllum wallisii. Acta Physiol. Plant. 2021, 43, 1-11. [CrossRef]

12. LeCun, Y.; Bengio, Y. Convolutional networks for images, speech, and time series. Handb. Brain Theory Neural Netw. 1995, $3361,1995$.

13. LeCun, Y.; Bengio, Y.; Hinton, G. Deep learning. Nature 2015, 521, 436-444. [CrossRef]

14. Li, W.; Xu, L.; Liang, Z.; Wang, S.; Cao, J.; Lam, T.C.; Cui, X. JDGAN: Enhancing generator on extremely limited data via joint distribution. Neurocomputing 2021, 431, 148-162. [CrossRef] 
15. Li, W.; Fan, L.; Wang, Z.; Ma, C.; Cui, X. Tackling mode collapse in multi-generator GANs with orthogonal vectors. Pattern Recognit. 2021, 110, 107646. [CrossRef]

16. Taheri-Garavand, A.; Nasiri, A.; Fanourakis, D.; Fatahi, S.; Omid, M.; Nikoloudakis, N. Automated In Situ Seed Variety Identification via Deep Learning: A Case Study in Chickpea. Plants 2021, 10, 1406. [CrossRef]

17. Nasiri, A.; Taheri-Garavand, A.; Fanourakis, D.; Zhang, Y.D.; Nikoloudakis, N. Automated Grapevine Cultivar Identification via Leaf Imaging and Deep Convolutional Neural Networks: A Proof-of-Concept Study Employing Primary Iranian Varieties. Plants 2021, 10, 1628. [CrossRef] [PubMed]

18. Hughes, D.; Salathé, M. An open access repository of images on plant health to enable the development of mobile disease diagnostics. arXiv 2015, arXiv:1511.08060.

19. Strange, R.N.; Scott, P.R. Plant disease: a threat to global food security. Annu. Rev. Phytopathol. 2005, 43, 83-116. [CrossRef] [PubMed]

20. Vilalta, R.; Drissi, Y. A perspective view and survey of meta-learning. Artif. Intell. Rev. 2002, 18, 77-95. [CrossRef]

21. Vanschoren, J. Meta-learning: A survey. arXiv 2018, arXiv:1810.03548.

22. Pan, S.J.; Qiang, Y. A Survey on Transfer Learning. IEEE Trans. Knowl. Data Eng. 2010, 22, 1345-1359. [CrossRef]

23. Snell, J.; Swersky, K.; Zemel, R.S. Prototypical networks for few-shot learning. arXiv 2017, arXiv:1703.05175.

24. Requeima, J.; Gordon, J.; Bronskill, J.; Nowozin, S.; Turner, R.E. Fast and flexible multi-task classification using conditional neural adaptive processes. Adv. Neural Inf. Process. Syst. 2019, 32, 7959-7970.

25. Bateni, P.; Goyal, R.; Masrani, V.; Wood, F.; Sigal, L. Improved few-shot visual classification. In Proceedings of the IEEE/CVF Conference on Computer Vision and Pattern Recognition, Seattle, WA, USA, 13-19 June 2020; pp. 14493-14502.

26. Lin, X.; Ye, M.; Gong, Y.; Buracas, G.; Basiou, N.; Divakaran, A.; Yao, Y. Modular Adaptation for Cross-Domain Few-Shot Learning. arXiv 2021, arXiv:2104.00619.

27. Cai, J.; Shen, S.M. Cross-domain few-shot learning with meta fine-tuning. arXiv 2020, arXiv:2005.10544.

28. Davis, J.V.; Kulis, B.; Jain, P.; Sra, S.; Dhillon, I.S. Information-theoretic metric learning. In Proceedings of the 24th International Conference on Machine Learning, Corvallis, OR, USA, 20-24 June 2007; pp. 209-216.

29. Vinyals, O.; Blundell, C.; Lillicrap, T.; Wierstra, D. Matching networks for one shot learning. Adv. Neural Inf. Process. Syst. 2016, $29,3630-3638$.

30. Triantafillou, E.; Zhu, T.; Dumoulin, V.; Lamblin, P.; Evci, U.; Xu, K.; Goroshin, R.; Gelada, C.; Swersky, K.; Manzagol, P.A.; et al. Meta-dataset: A dataset of datasets for learning to learn from few examples. arXiv 2019, arXiv:1903.03096.

31. Russakovsky, O.; Deng, J.; Su, H.; Krause, J.; Satheesh, S.; Ma, S.; Huang, Z.; Karpathy, A.; Khosla, A.; Bernstein, M.; et al. Imagenet large scale visual recognition challenge. Int. J. Comput. Vis. 2015, 115, 211-252. [CrossRef]

32. Lake, B.M.; Salakhutdinov, R.; Tenenbaum, J.B. Human-level concept learning through probabilistic program induction. Science 2015, 350, 1332-1338. [CrossRef]

33. Maji, S.; Rahtu, E.; Kannala, J.; Blaschko, M.; Vedaldi, A. Fine-grained visual classification of aircraft. arXiv 2013, arXiv:1306.5151.

34. Wah, C.; Branson, S.; Welinder, P.; Perona, P.; Belongie, S. The Caltech-Ucsd Birds-200-2011 Dataset; California Institute of Technology: Pasadena, CA, USA, 2011.

35. Cimpoi, M.; Maji, S.; Kokkinos, I.; Mohamed, S.; Vedaldi, A. Describing textures in the wild. In Proceedings of the IEEE Conference on Computer Vision and Pattern Recognition, Columbus, OH, USA, 23-28 June 2014, pp. 3606-3613.

36. Wheatley, G. Quick Draw; Mathematics Learning: Bethany Beach, DE, USA, 2007.

37. Schroeder, B.; Cui, Y. Fgvcx Fungi Classification Challenge 2018. Available online: github.com/visipedia/fgvcx_fungi_comp (accessed on 14 July 2021).

38. Nilsback, M.E.; Zisserman, A. Automated flower classification over a large number of classes. In Proceedings of the 2008 Sixth Indian Conference on Computer Vision, Graphics \& Image Processing, Bhubaneswar, India, 16-19 December 2008; pp. 722-729.

39. Houben, S.; Stallkamp, J.; Salmen, J.; Schlipsing, M.; Igel, C. Detection of traffic signs in real-world images: The German Traffic Sign Detection Benchmark. In Proceedings of the 2013 International Joint Conference on Neural Networks (IJCNN), Dallas, TX, USA, 4-9 August 2013; pp. 1-8.

40. Lin, T.Y.; Maire, M.; Belongie, S.; Hays, J.; Perona, P.; Ramanan, D.; Dollár, P.; Zitnick, C.L. Microsoft coco: Common objects in context. In Proceedings of the European Conference on Computer Visio, Zurich, Switzerland, 6-12 September 2014 ; pp. 740-755.

41. Thapa, R.; Zhang, K.; Snavely, N.; Belongie, S.; Khan, A. The Plant Pathology Challenge 2020 data set to classify foliar disease of apples. Appl. Plant Sci. 2020, 8, e11390. [CrossRef]

42. He, K.; Zhang, X.; Ren, S.; Sun, J. Deep residual learning for image recognition. In Proceedings of the IEEE Conference on Computer Vision and Pattern Recognition, Las Vegas, NV, USA, 27-30 June 2016; pp. 770-778.

43. Finn, C.; Abbeel, P.; Levine, S. Model-agnostic meta-learning for fast adaptation of deep networks. In Proceedings of the International Conference on Machine Learning, PMLR, Sydney, Australia, 6-11 August 2017; pp. 1126-1135.

44. Perez, E.; Strub, F.; De Vries, H.; Dumoulin, V.; Courville, A. Film: Visual reasoning with a general conditioning layer. In Proceedings of the AAAI Conference on Artificial Intelligence, New Orleans, LA, USA, 2-7 February 2018 ; Volume 32.

45. Li, W.; Liu, X.; Liu, J.; Chen, P.; Wan, S.; Cui, X. On improving the accuracy with auto-encoder on conjunctivitis. Appl. Soft Comput. 2019, 81, 105489. [CrossRef]

46. Joachims, T. Making Large-Scale SVM Learning Practical. Technical Report. 1998. Available online: https://www.econstor.eu/ handle/10419/77178 (accessed on 9 March 2021). 
47. Li, W.; Wang, L.; Xu, J.; Huo, J.; Gao, Y.; Luo, J. Revisiting local descriptor based image-to-class measure for few-shot learning. In Proceedings of the IEEE/CVF Conference on Computer Vision and Pattern Recognition, Long Beach, CA, USA, 15-20 June 2019; pp. 7260-7268.

48. Zhang, Z.; Sabuncu, M.R. Generalized cross entropy loss for training deep neural networks with noisy labels. In Proceedings of the 32nd Conference on Neural Information Processing Systems (NeurIPS), Montreal, QC, Canada, 3-8 December 2018.

49. Kingma, D.P.; Ba, J. Adam: A method for stochastic optimization. arXiv 2014, arXiv:1412.6980.

50. Selvaraju, R.R.; Cogswell, M.; Das, A.; Vedantam, R.; Parikh, D.; Batra, D. Grad-cam: Visual explanations from deep networks via gradient-based localization. In Proceedings of the IEEE International Conference on Computer Vision, Venice, Italy, 22-29 October 2017; pp. 618-626.

51. Chatzistathis, T.; Fanourakis, D.; Aliniaeifard, S.; Kotsiras, A.; Delis, C.; Tsaniklidis, G. Leaf Age-Dependent Effects of Boron Toxicity in Two Cucumis melo Varieties. Agronomy 2021, 11, 759. [CrossRef]

52. Taheri-Garavand, A.; Mumivand, H.; Fanourakis, D.; Fatahi, S.; Taghipour, S. An artificial neural network approach for noninvasive estimation of essential oil content and composition through considering drying processing factors: A case study in Mentha aquatica. Ind. Crop. Prod. 2021, 171, 113985. [CrossRef] 